\title{
About a rare cause of intestinal perforation
}

\author{
Mona Mlika ${ }^{1}$, Heifa Azouz ${ }^{1}$, Inès Chelly ${ }^{1}$, Sofiène Ayadi ${ }^{2}$, Wael Rbai ${ }^{2}$, Nidhameddine Kchir ${ }^{1}$, Slim \\ Haouet $^{1}$, Mohamed Moncef Zitouna ${ }^{1}$
}

\footnotetext{
${ }^{1}$ Department of Pathology, La Rabta Hospital, Bab Saadoun, Tunis, Tunisia;

${ }^{2}$ Department of Surgery, La Rabta Hospital, Bab Saadoun, Tunis, Tunisia.

Email: mlika.zorgati.mona@hotmail.com
}

Received 23 May 2011; revised 26 May 2011; accepted 12 August 2011.

\begin{abstract}
Background: Periarteritis nodosa (PAN) is a necrotizing systemic vasculitis involving medium-sized arteries and rarely the small-sized ones. It affects various organs and is generally revealed by different non specific organs. The aim of the authors is to report a rare revealing manifestation of this disease which is the perforation of the small bowel and to highlight the most important histological features. Case report: The authors describe the case of a 48-year-old Caucasian woman, without a particular past medical history, who presented an acute surgical abdomen. A surgical exploration was decided and showed a necrosis of the small bowel and the appendix. A segmental ileectomy was performed. The histological examination of the specimen showed small vasculitis-affected with fibroid necrosis dealing to the diagnosis of intestinal perforation caused by a PAN was retained. Post-operatively, the patient was put on immunosuppressive therapy and presented a prolonged healing process and was discharged from the hospital 20 days after her admission. Conclusion: Although it is rare for PAN to be revealed by small bowel perforation, it should be kept in mind when facing ischemic change of the small bowel.
\end{abstract}

Keywords: Periarteritis Nodosa; Intestinal Perforation; Vasculitis

\section{INTRODUCTION}

Periarteritis nodosa (PAN) affects various organs and involves the gastrointestinal tract in up to $50 \%$ in autopsy series. Perforation of the small bowel is a rare complication and can reveal the disease in exceptional cases. This report describes the case of a 48-year-old woman who developed a perforation of the small bowel with ischemic change due to PAN.

\section{CASE REPORT}

A 48-year-old Caucasian woman, without a particular past medical history, presented a two-week lasting myalgias and multiple mononeuropathies. A muscular biopsy was performed and histological examination concluded to a non specific systemic vasculitis. After three weeks, she presented an acute surgical abdomen. Physical examination showed a reduced bowel sounds with a distended abdomen and a diffuse tenderness. Laboratory tests showed a white cell count of $11000 / u l$, a C-reactive protein level of $22 \mathrm{mg} / \mathrm{dl}$, an erythrocyte sedimentation rate of $41 \mathrm{~mm} / \mathrm{h}$. Urinalysis showed 1+ proteinuria. Hepatitis B surface antigen was negative and Antineutrophil cytoplasmic autoantibody (ANCA) was not measured on admission. A surgical exploration was decided. The laparotomy showed a necrosis of the small bowel and the appendix. A segmental ileectomy was performed. The histological examination of the specimen showed small vasculitis-affected vessels including an inflammatory infiltrate leading to the complete destruction and thrombosis of their lumen. The inflammatory infiltrate was made of polymorphonuclear leukocytes and a variable number of lymphocytes, macrophages and eosinophils. Fibroid necrosis was present with a disruption of the lastic laminae (Figures 1 and 2).

According to the histological findings, the diagnosis of intestinal perforation caused by a peri-arteritis nodosa was retained. Post-operatively, immunosuppressive therapy was initiated with the intravenous administration of prednisone $(1 \mathrm{mg} / \mathrm{kg} / \mathrm{d})$ and cyclophosphamide $(1 \mathrm{mg} / \mathrm{Kg} / \mathrm{d})$. The patient presented a prolonged healing process and was discharged from the hospital 20 days after her admission.

\section{DISCUSSION}

Polyarteritis nodosa (PAN) is a necrotizing systemic vasculitis involving medium-sized arteries and rarely the small-sized ones. Digestive manifestations are rather frequent in PAN in comparison with the other vasculitis and 


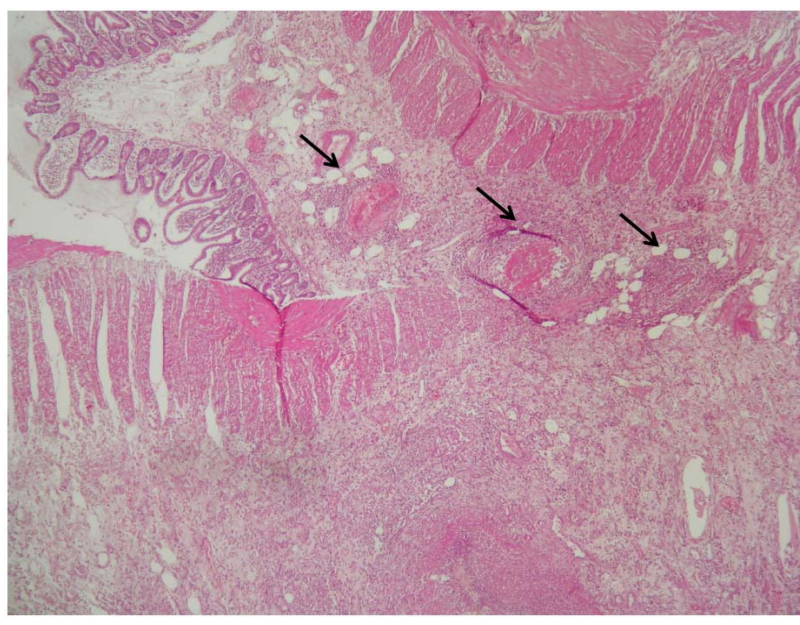

Figure 1. Intestinal wall comprising destroyed middle-sized vessels with thrombosis of their lumen $(\mathrm{HE} \times 100)$.

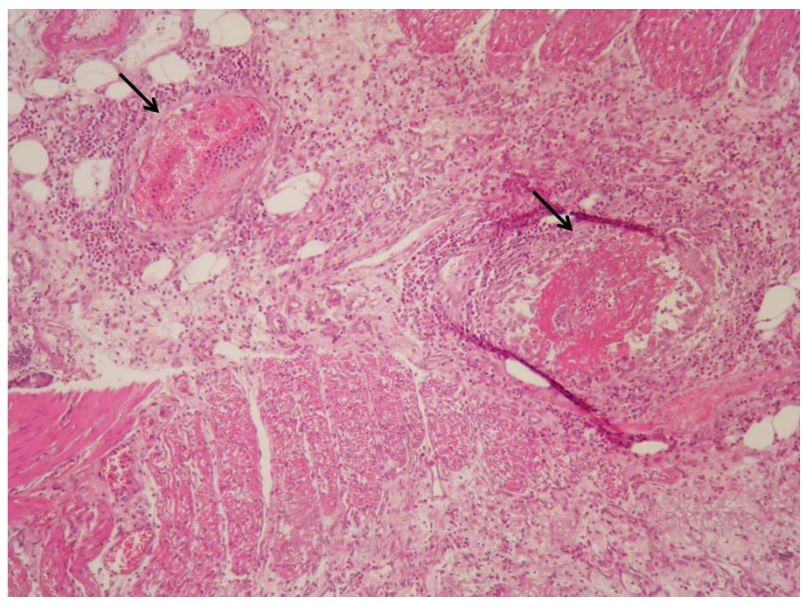

Figure 2. Fibroid necrosis with disruption of the lastic lamina $(\mathrm{HE} \times 400)$.

account for $14 \%$ to $65 \%$ [1]. In opposition to the frequent digestive manifestations, intestinal perforation is a rare complication mentioned in few reports. Digestive manifestations are rather frequent in PAN but they reveal the disease only in $16 \%$ of the cases. Digestive symptoms are non specific consisting in abdominal pain, diarrhea, nausea and digestive haemorrhage. All these symptoms account respectively for $30 \%$ to $95 \%$, $21 \%$ to $50 \%, 17 \%$ and $8 \%$ to $45 \%$ of the cases [2]. The location in the small bowel is quite frequent but relevant because of the multiplicity of perforations and ischemia. These complications account for $2 \%$ to $40 \%$. Mesenteric vasculitis preferentially affects the superior mesenteric artery supply, although the inferior mesenteric artery also may be involved [3]. The ileum and jejunum are the most frequent locations ( $80 \%$ to $85 \%$ ). Unlike the other vasculitides such as microscopic polyarteritis or Wegener's granulomatosis, polyarteritis nodosa is not typically as- sociated with antineutrophil cytoplasmic antibodies (ANCA) [4]. Radiological examination, especially digestive arteriography, is helpful in diagnosing arterial micro-aneurysms which are rarely revealed by CT-scan. Non invasive imaging is an important diagnostic modality. CT scan is the first option allowing to distinguish thrombo-embolic disease from vasculitis and occlusive from benign vasculitis [3]. Angiography shows generally aneurysms of the mesenteric artery. This exam has a $80 \%$ true positive rate in patients with PAN [3]. It can show symmetrical bowel thickening, target signs and vascular engorgement and haziness [3]. All these findings are non specific. When vasculitis presents initially with bowel ischemia, the diagnosis is often established postoperatively after pathologic examination. If the patient is young, without predisposing factors such as cardiac arythmia, an atherosclerotic disease, vasculitis should be considered early in the differential diagnosis [3]. Macroscopic examination of the affected intestine may show multiple involvement anatomic regions not corresponding to a single vascular supply and evidence of ischemic regions of varying chronologic appearance [3] Histopathologic examination demonstrates transmural inflammation of the arterial wall with a heavy infiltrate of polymorphs, eosinophils, and mononuclear cells. This inflammation is frequently accompanied by fibrinoid necrosis of the inner half of the vessel wall. Thrombus can be observed. The etiology of polyarteritis nodosa is unknown, but many cases occur in individuals infected with hepatitis B virus (HBV), and, occasionally, hepatitis C virus [2]. Cases associated with hepatitis $B$ are immune complex mediated. HBV surface antigen may be found in active lesions, implicating a role in the pathogenesis. HBV-associated cases usually manifest in the first six months of infection and may be the presenting sign of the infection [4]. The classification and the management of the systemic vasculitis remain debated and non consensual; in fact, despite of the American College of Rheumatology classification and the Chapel Hill Consensus nomenclature, many definitions remain unclear. For example, a distinction between PAN and MPA remain difficult and many researches were made in order to discriminate between these 2 entities such as the French Vasculitis study group [5]. This distinction may be confusing, so the features of each condition need to be more accurately defined. Differentiation between PAN and MPA cannot be based only on histological criteria. Clinical manifestations (especially lung and kidney involvement), biologic signs (antineutrophil cytoplasmic antibodies and hepatitis B and C infection), and angiographic data must be taken into consideration. The management of this vasculitis is based on medical treatment including aggressive antiinflamematory and immunosuppressive tr- 
eatment [3]. Surgical treatment should be avoided and performed only in complicated cases. In fact, because of the lesions of vasculitis, many cases of leakage of anastomosis and healing problems are reported. Our patient presented a prolonged healing process. The prognosis of the digestive complications is very worse. In fact, mortality reaches $25 \%$ to $75 \%$ in PAN with an acute surgical abdomen [3].

\section{REFERENCES}

[1] Guillevin, L., Lhote, F., Gayraud, M., et al. (1996) Prognostic factors in polyarteritis nodosa and Churg-Strauss syndrome. A prospective study in 342 patients. Medicine, 75, 17-28. doi:10.1097/00005792-199601000-00003
[2] Pagnoux, C. and Guillevin, L. (2006) Digestive manifestations of systemic vasculitis. La Revue de Médecine Interne, 27, 56-58. doi:10.1016/j.revmed.2006.03.019

[3] Passam, F.H., Diamantis, I.D., Perisinaki, G., et al. (2004) Intestinal ischemia as the first manifestation of vasculitis. Seminars in Arthritis and Rheumatism, 34, 431-441. doi:10.1016/j.semarthrit.2003.12.004

[4] Kallenberg, C.G., Brouwer, E., Weening, J. and Cohen, J.W. (1994) Anti-neutrophil cytoplasmic antibodies: Current diagnostic and pathophysiological potential. Kidney International, 46, 1-15. doi:10.1038/ki.1994.239

[5] Henegar, C., Pagnoux, C., Puéchal, X., et al. (2008) A paradigm of diagnostic criteria for polyarteritis nodosa. Arthritis and Rheumatism, 58, 1528-1538. doi:10.1002/art.23470 